\title{
Cezanne predicts progression and adjuvant TACE response in hepatocellular carcinoma
}

\author{
Jia-hong Wang ${ }^{1,2,7}$, Xiao-ping Zhong ${ }^{1,7}$, Yong-fa Zhang ${ }^{3,4,5,7}$, Xiao-liang Wu ${ }^{6}$, Shao-hua Li ${ }^{1}$, Pei-en Jian', Yi-hong Ling ${ }^{1}$, Ming Shi ${ }^{1}$, \\ Min-shan Chen ${ }^{1}$, Wei Wei, ${ }^{*, 1,8}$ and Rong-ping Guo ${ }^{\star, 1,8}$
}

We have previously reported that Cezanne could be a prognostic biomarker for survival in hepatocellular carcinoma (HCC) patients. However, the role of Cezanne genes in HCC cells and its response to postoperative adjuvant transcatheter arterial chemoembolization (TACE) in HCC patients remains unknown. In this study, Cezanne expression was detected in human HCC using real-time PCR, western blot and immunohistochemistry. The function of Cezanne in HCC cells was determined by Transwell invasion assays and nude mice metastasis assay. The response of Cezanne in patients who received adjuvant TACE after hepatectomy was evaluated. Functional study demonstrated that interference of Cezanne expression promoted the migration and invasion of HCC cells in vitro and boosted metastasized HCC formation in mice. Upregulation of Cezanne diminished the adhesion and migration of hepatoma cells. Further study indicated that Cezanne might inhibit invasion of HCC cells by inducing epithelialmesenchymal transition (EMT). In addition, patients with low Cezanne expression had significant improvement in prognosis after receiving adjuvant TACE. In contrast, patients with high Cezanne expression had a poorer response to adjuvant TACE. Moreover, Cezanne status was associated with response to adjuvant TACE in patients subgroup stratified by vascular invasion, tumor size and tumor number. In conclusion, Cezanne may be a novel antioncogene that has a pivotal role in the invasion of HCC and contribute to the selection of patients who may benefit from adjuvant TACE to prevent recurrence.

Cell Death and Disease (2017) 8, e3043; doi:10.1038/cddis.2017.428; published online 7 September 2017

Hepatocellular carcinoma (HCC) is one of the most common solid tumors and prevalent fatal cancers worldwide, especially in East Asia and Sub-Saharan Africa. ${ }^{1,2}$ HCC generally arises in cirrhotic livers and has a poor prognosis because of the high incidence of disease recurrence after curative treatment. ${ }^{3,4}$ Therefore, the prevention of recurrence constitutes one of the most important challenges in improving surgical efficacy. Systemic chemotherapy and transcatheter arterial chemoembolization (TACE) are the commonly used adjuvant managements in preventing recurrence and prolonging the survival of patients after hepatectomy. However, although a number of controlled and uncontrolled studies have been performed with most classes of chemotherapeutic agents, no single or combination chemotherapy regimen is significantly effective in $\mathrm{HCC}^{5,6}$ and only a few of them have shown improved response rates. ${ }^{7}$ Several studies reported that patients with large tumors, venous invasion or intrahepatic metastasis had been recommended to receive TACE $1-2$ months after resection. ${ }^{8-10}$ However, deterioration of liver function after TACE may negatively affect the patients' prognosis and liver function. ${ }^{11}$ In addition, survival may vary widely among HCC patients with the same clinicopathologic features that is most likely attributable to the heterogeneity of the biological behavior of tumor cells. ${ }^{12,13}$ Therefore, screening the biomarkers for better valuation of recurrence and prognosis of $\mathrm{HCC}$ could revolutionize treatment of HCC and is the key goal of modern personalized medicine. ${ }^{14}$

Cezanne is a member of the A20 family of deubiquitinating enzymes. ${ }^{15}$ Similar to A20, Cezanne has been shown to inhibit NF- $K B$ pathway by deconjugating K63-polyubiquitin chains from RIP-1 and TRAF6, ${ }^{15,16}$ suggesting that it may have roles in inhibition of cancer progression. Our previous results found that Cezanne was associated with tumor size, vascular invasion and satellite nodule in HCC. Cezanne may act as a potential prognostic biomarker for survival in HCC patients. ${ }^{17}$ Therefore, we hypothesized that the effect of postoperative adjuvant TACE on survival in patients with low Cezanne expression may differ from those with high Cezanne expression. In this study, we evaluated the role of Cezanne genes in $\mathrm{HCC}$ and its response to postoperative adjuvant TACE in HCC patients.

\footnotetext{
${ }^{1}$ Department of Hepatobilliary Oncology, Sun Yat-sen University Cancer Center; State Key Laboratory of Oncology in South China; Collaborative Innovation Center for Cancer Medicine, Guangzhou, China; ${ }^{2}$ Department of Abdominal Surgery, Affiliated Cancer Hospital \& Institute of Guangzhou Medical University, Guangzhou, Guangdong, China; ${ }^{3}$ Department of Liver Surgery, Fudan University Shanghai Cancer Center, Shanghai, China; ${ }^{4}$ Department of Oncology, Shanghai Medical College, Fudan University, Shanghai, China; ${ }^{5}$ Department of Laboratory, Fudan University Shanghai Cancer Center, Shanghai, China and ${ }^{6}$ Department of Oncology, Guizhou Provincial People's Hospital, Guiyang, China

*Corresponding author: W Wei or R-p Guo, Department of Hepatobilliary Oncology, Sun Yat-sen University Cancer Center; State Key Laboratory of Oncology in South China; Collaborative Innovation Center for Cancer Medicine, 651 Dongfeng Road East, Guangzhou 510060, China. Tel: +86 20 87343114; Fax: +86-20-87343114; E-mail: weiwei@sysucc.org.cn or guorp@sysucc.org.cn

${ }^{7}$ These authors contributed equally to this work.

${ }^{8}$ These authors jointly supervised this work.

Received 30.5.17; revised 18.7.17; accepted 20.7.17; Edited by A Stephanou
} 


\section{Results}

Cezanne expression is associated with HCC cell invasion. Our previous results had reported that Cezanne has a pivotal role in tumor progression and prognosis, and may act as a potential prognostic biomarker for survival in HCC patients. We further investigated the role of Cezanne in tumor cell migration and invasion in HCC cells. Transwell invasion assay revealed that the ability of cell motility and invasion increased in shCezanne-SK-Hep1 cells as compared with those with shCon-SK-Hep1 cells (both $P<0.001$, Figures 1a and $\mathrm{c}$ ), whereas the ability of cell motility and invasion decreased in Cezanne-SMMC-7721 cells as compared with those with Vector-SMMC-7721 cells (both $P<0.001$, Figures $1 \mathrm{~b}$ and $\mathrm{d}$ ). To evaluate the in vivo effects of Cezanne on tumor metastasis, two groups of eight mice each were injected intravenously in the tail vein with shCon-SK-Hep1 or shCezanne-SK-Hep1 cells, respectively. After 8 weeks, the mice were killed and the metastatic nodules at the lung surfaces were counted. There were larger numbers of metastatic nodules at the surface of the lungs of mice injected with the shCezanne-SK-Hep1 cells than those with the shCon-SK-Hep1 cells $(P<0.001$, Figure 1e). Hematoxylin and eosin (H\&E) staining confirmed that the nodules on the surfaces of mice lungs were metastatic tumors (Figure 1e). CCK-8 assays revealed that the SK-Hep1 with low expression of Cezanne showed more proliferation capacity compared with the control cells. In contrast, overexpression of Cezanne could inhibit the proliferation of SMMC-7721 (Supplementary Figure S1).
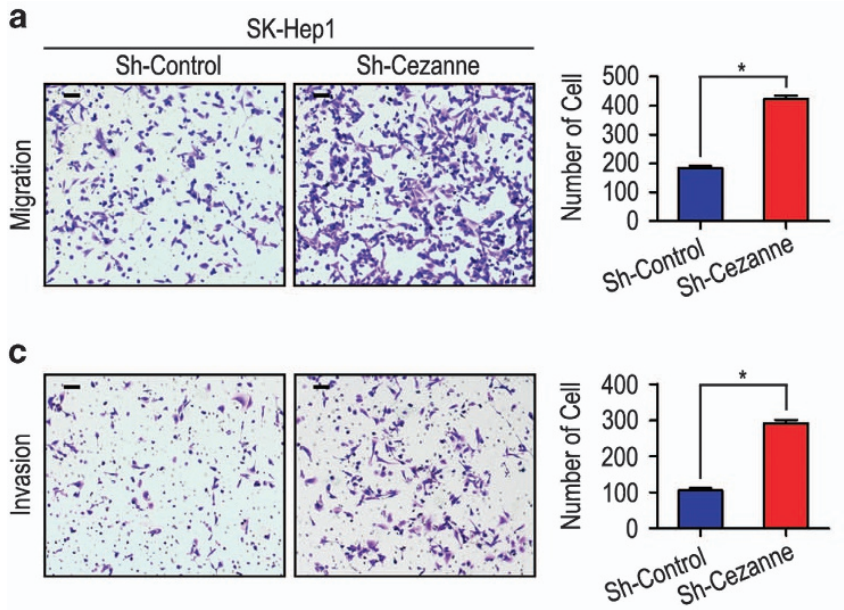

As epithelial-mesenchymal transition (EMT) is one of the key events in tumor invasion and metastasis, the effect of Cezanne on EMT markers was analyzed. The relationship of Cezanne and EMT markers such as E-cadherin and vimentin was confirmed by IHC assays in serial sections of HCC tissues (Figures $2 \mathrm{a}$ and b). Moreover, our western blot and RT-PCR results showed that Cezanne expression was positively correlated with E-cadherin and negatively correlated with vimentin expression (Figures 2c and d). Furthermore, decreased expression of E-cadherin and increased expression of vimentin was observed in tumors induced by shCezanne-SK-Hep1 cells compared with those induced by shCon-SK-Hep1 cells (Figure 2e).

Prognostic significance of postoperative adjuvant TACE within the Cezanne level. Adjuvant TACE is one of the most used methods to prevent tumor recurrence. However, the curative effect of adjuvant TACE remains unclear. In our results, we found that adjuvant TACE after surgery did not improve the overall survival (OS) and time to recurrence (TTR) rates of the whole study population (Figure 3a). Because Cezanne may predict the prognosis (OS and TTR) of $\mathrm{HCC}$, we next investigated whether Cezanne expression in the tumor was correlated with the response of patients to adjuvant TACE therapy. Patients with low Cezanne expression in tumors had significant improvement in the OS and TTR after receiving adjuvant therapy with postoperative TACE as compared with those without adjuvant TACE (Figure $3 b$ ). In contrast, patients with high Cezanne

\section{b}
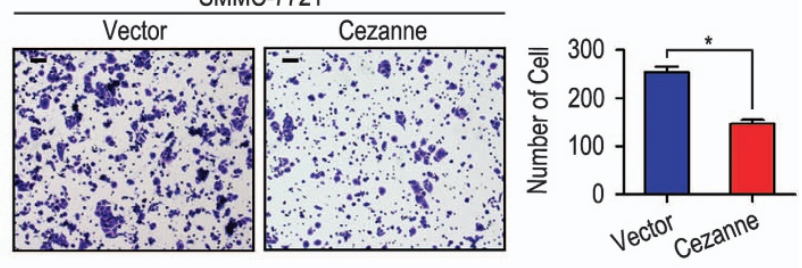

d
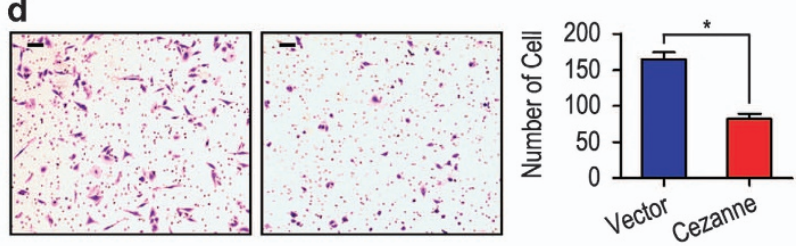

e

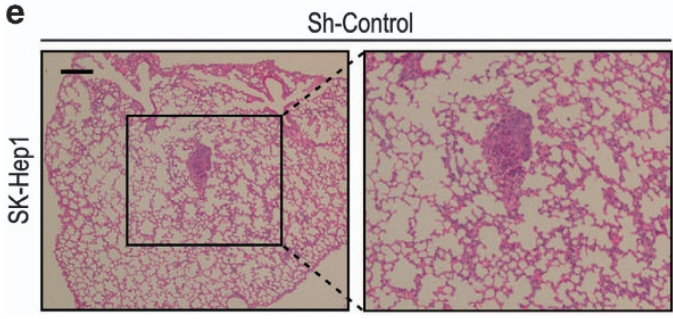

Sh-Cezanne

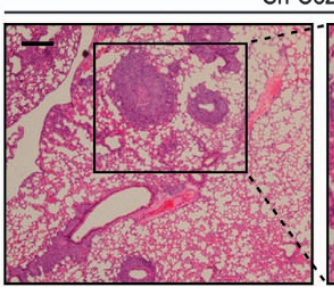

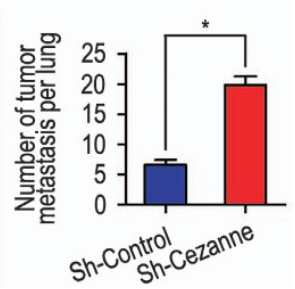

Figure 1 Cezanne inhibits cell migration and invasion in HCC cell lines. (a and c) Cezanne was downregulated in SK-Hep1 cells and promoted cell migration and invasion. (b and d) Cezanne was upregulated in SMMC-7721 cells and inhibited cell migration and invasion. (e) Lung morphology and H\&E staining of nude mice inoculated with SK-Hep1 sh-Cezanne or control cells via tail vein. The number of lung metastatic foci in each group were also calculated. ${ }^{\star} P<0.05$. The scale bar represents $50 \mu \mathrm{m}$ 
a
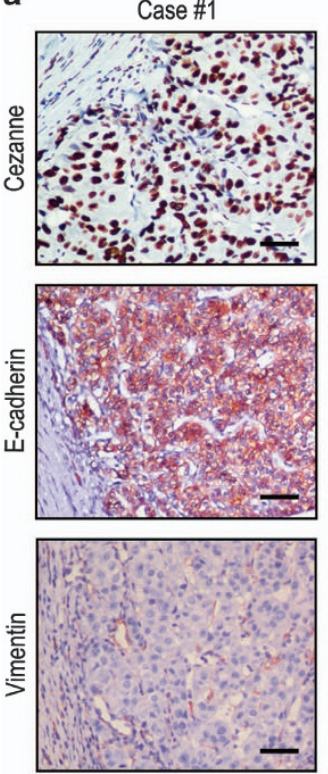

C

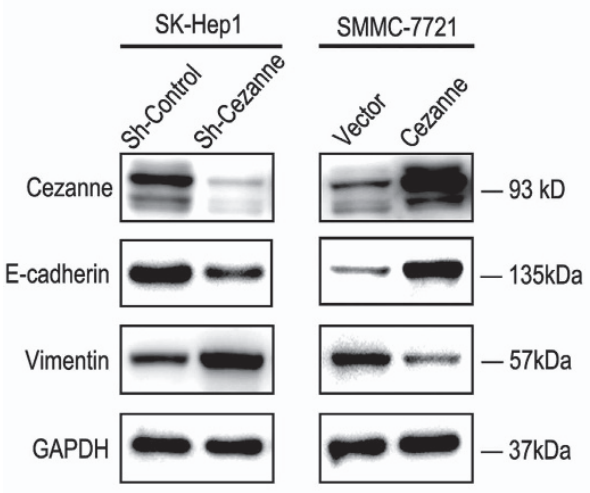

b
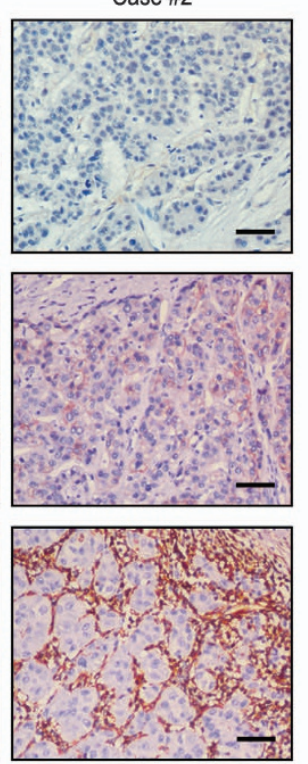

d
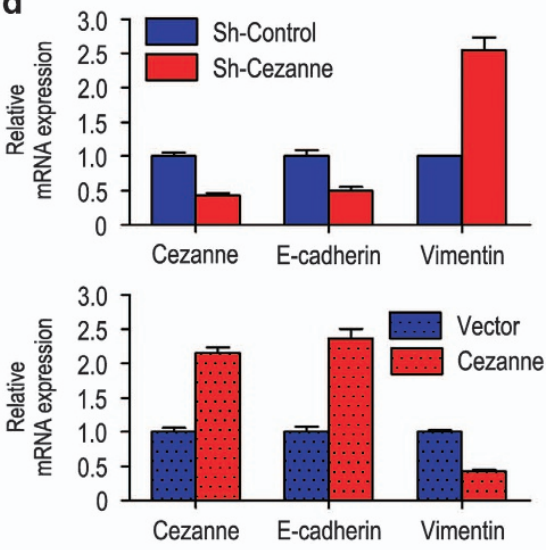

e
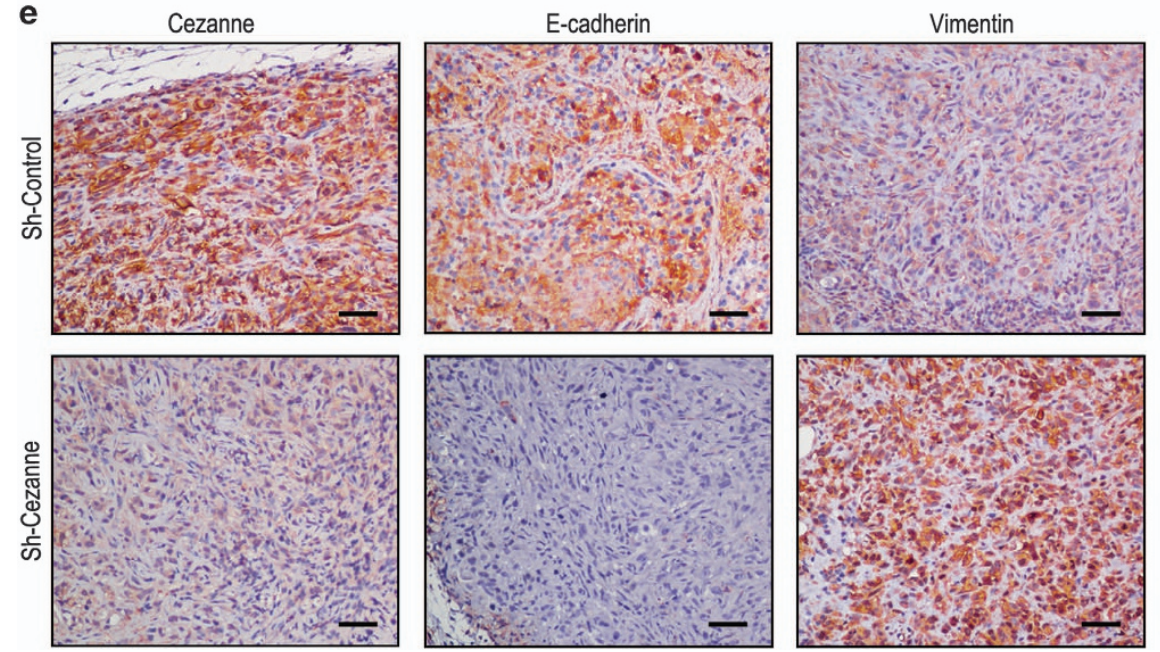

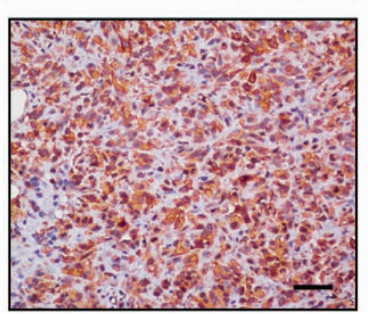

Figure 2 Cezanne expression level correlated with the expression of epithelial-mesenchymal transition (EMT) markers. (a) Serial sections of human HCC tissues were subjected to IHC staining with antibodies against Cezanne, E-cadherin and vimentin. In case 1, high expression of Cezanne in HCC tissues was accompanied by elevated $\mathrm{E}$-cadherin and the absence of vimentin. In case 2, low expression of Cezanne was accompanied by the absence of E-cadherin and elevated vimentin. The scale bar represents $50 \mu \mathrm{m}$. (b) Cezanne expression was positively correlated with E-cadherin expression and negatively associated with vimentin expression. (c and d) Transfecting siRNAs against Cezanne decreased Cezanne expression, downregulated E-cadherin and upregulated vimentin. Overexpression of Cezanne upregulated E-cadherin and downregulated vimentin. (e) Representative images of IHC staining of mice xenografts of SK-Hep1 sh-Cezanne and control cells. The scale bar represents $50 \mu \mathrm{m}$ 
a

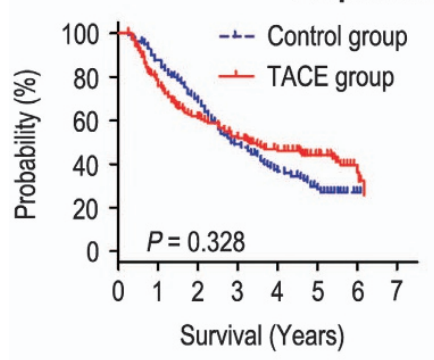

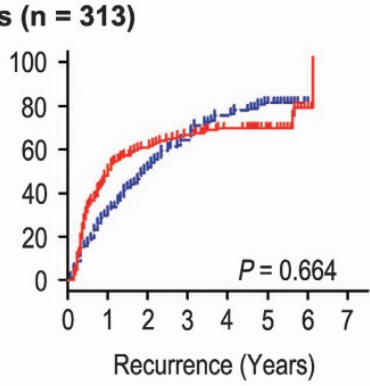

b
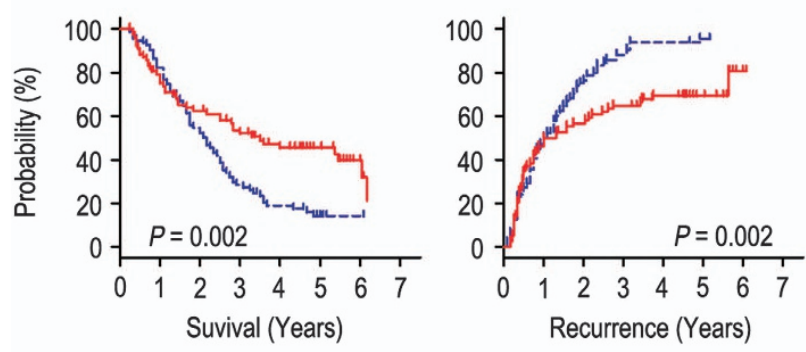

C
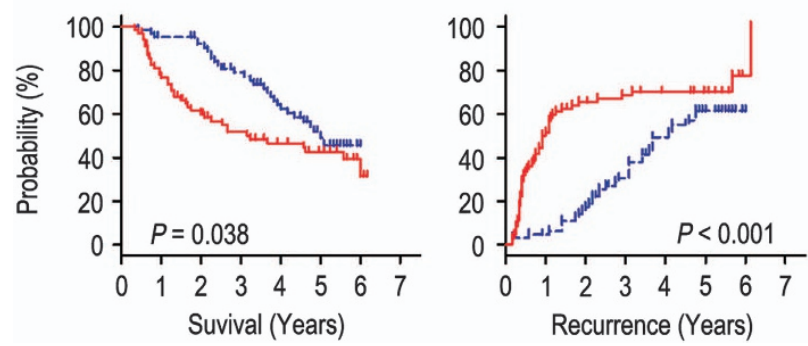

Figure 3 Prognostic significance of postoperative adjuvant TACE according to Cezanne expression. Kaplan-Meier analysis of the correlation between adjuvant TACE therapy and prognosis in all HCC patients (a). Kaplan-Meier analysis of the correlation between adjuvant TACE therapy and OS/TTR in patients with low Cezanne expression (b) and high Cezanne expression (c)

expression in tumors had a poorer response to adjuvant TACE (Figure 3c). The median values of OS times for adjuvant TACE group and Control group were 25.5 and 49.0 months, whereas the median values of TTR times were 11.5 and 41.0 months (Figure $3 c$ ). In addition, we also found that the expression of EMT relevant markers (E-cadherin and vimentin) had no correlation with the response of patients to adjuvant TACE therapy (data not show).

In addition, we used Cox proportional hazards regression to further evaluate the correlation between Cezanne expression and response to adjuvant TACE in HCC patients. In the low Cezanne-expressed HCC patients, adjuvant TACE, liver cirrhosis and tumor size were significantly associated with OS (Table 1). Furthermore, Kaplan-Meier analysis demonstrated that HBsAg, liver cirrhosis, lager tumor size and without adjuvant TACE were correlated with shorter TTR in low Cezanne-expressed patients (Table 2). Multivariate Cox regression analysis revealed that adjuvant TACE was an independent predictor for OS $(\mathrm{HR}=0.536,95 \% \mathrm{Cl}=0.362$ $0.794, P=0.002)$ and TTR $(\mathrm{HR}=0.568,95 \% \mathrm{Cl}=0.397-$ 0.812, $P=0.002$ ) in low Cezanne-expressed patients.
However, adjuvant TACE could not act as a potential prognostic biomarker for survival in high Cezanne-expressed patients.

Cezanne predicts response to adjuvant TACE in clinical subgroups. The HCC patients with vascular invasion, larger tumor ( $>5 \mathrm{~cm}$ in diameter) or multiple tumors are recommended to receive TACE $1-2$ months after resection. ${ }^{8,9,10}$ Our findings had similar results with previous study. We found that adjuvant TACE could improve the prognosis (OS and TTR) of HCC patients with vascular invasion (Supplementary Figure S2b) and larger tumor (Supplementary Figure S2d), whereas there were no significant differences in patients with no vascular invasion (Supplementary Figure S2a), small tumor size (Supplementary Figure S2c) and single or multiple tumors (Supplementary Figures $\mathrm{S} 2 \mathrm{e}$ and f). Our previous study confirmed that Cezanne could be a potential prognostic biomarker for prognosis in HCC patients. We therefore evaluated the discriminative power of Cezanne levels within the vascular invasion, tumor size or tumor number status on postoperative adjuvant TACE. In patients with no vascular invasion, small tumor or single tumor, high Cezanne expression had a bad response on adjuvant TACE, whereas low Cezanne expression had a better median TTR than control group (Figures $4 \mathrm{a}, \mathrm{c}$ and e). On the contrary, in the patients with vascular invasion, larger tumor or multiple tumor, high Cezanne expression had no response on adjuvant TACE, whereas adjuvant TACE was associated with significant improvement in TTR of HCC patients with low Cezanne expression (Figures $4 b$, $d$ and f). Moreover, in the subgroups of no vascular invasion, small tumor or single tumor patients, those with Cezanne overexpression had shorter OS (Supplementary Figure S3a) or no response (Supplementary Figures $\mathrm{S} 3 \mathrm{c}$ and $\mathrm{d}$ ) on adjuvant TACE, whereas those with low expression of Cezanne had a better OS than control group (Supplementary Figures S3a, c and e). In contrast, in the subgroups of vascular invasion, larger tumor or multiple tumor patients, the high Cezanne expression patients had no difference in OS irrespective of whether they received TACE or not, whereas adjuvant TACE could improve the OS in low Cezanne expression patients with vascular invasion or larger tumor (Supplementary Figures S3b, d and f).

\section{Discussion}

Because of high incidence of recurrence and metastasis after hepatectomy, the long survival of HCC patients remains unsatisfactory. In addition, intrahepatic metastasis was thought to have a close relation with the postoperative recurrence. ${ }^{18}$ Adjuvant TACE could inhibit residual tumor after resection of HCC. However, it is also reported that the benefits of adjuvant TACE depended on the selection of patients. ${ }^{9}$ Adjuvant TACE could prolong the survival of patients who had high risks of recurrence in remnant liver because of therapeutic actions on the residual tumor. ${ }^{19,20}$ Thus, the identification of new predictive biomarkers of HCC invasion and prognosis is critical. In this study, we explored the oncogenicity of Cezanne in the progression of HCC, and 
Table 1 Univariate and multivariate analysis of OS in different Cezanne expressions of HCC patients

\begin{tabular}{|c|c|c|c|c|c|c|c|c|}
\hline \multirow[t]{3}{*}{ Variables } & \multirow{3}{*}{$\frac{\text { Univariate }}{P \text {-value }}$} & \multirow{2}{*}{\multicolumn{3}{|c|}{$\begin{array}{c}\text { Low Cezanne expression } \\
\text { Multivariate }\end{array}$}} & \multirow{3}{*}{$\frac{\text { Univariate }}{P \text {-value }}$} & \multirow{2}{*}{\multicolumn{3}{|c|}{$\begin{array}{c}\text { High Cezanne expression } \\
\text { Multivariate }\end{array}$}} \\
\hline & & & & & & & & \\
\hline & & $P$-value & HR & $95 \% \mathrm{Cl}$ & & $P$-value & HR & $95 \% \mathrm{Cl}$ \\
\hline Gender (female versus male) & NS & NS & & & NS & NS & & \\
\hline Age, years $(\leqslant 50$ versus $>50)$ & NS & NS & & & NS & NS & & \\
\hline AFP $(\mathrm{ng} / \mathrm{ml})(\leqslant 400$ versus $>400)$ & NS & NS & & & NS & NS & & \\
\hline HBsAg (negative versus positive) & NS & NS & & & 0.001 & 0.003 & 0.307 & $0.143-0.662$ \\
\hline GGT $(\mathrm{U} / \mathrm{l})(\leqslant 50$ versus $>50)$ & NS & NS & & & $<0.001$ & NS & & \\
\hline Liver cirrhosis (no versus yes) & $<0.001$ & 0.001 & 2.504 & $1.465-4.282$ & NS & NS & & \\
\hline Tumor size $(\mathrm{cm})(\leqslant 5$ versus $>5)$ & 0.014 & 0.001 & 1.928 & $1.310-2.836$ & $<0.001$ & 0.021 & 1.976 & $1.108-3.525$ \\
\hline Tumor number (single versus multiple) & NS & NS & & & $<0.001$ & 0.026 & 1.894 & $1.077-3.328$ \\
\hline Satellite nodule (no versus yes) & NS & NS & & & NS & NS & & \\
\hline Tumor capsule (no/ incomplete versus complete) & NS & NS & & & NS & NS & & \\
\hline Tumor differentiation (I-II versus III-IV) & NS & NS & & & NS & NS & & \\
\hline Vascular invasion (no versus yes) & NS & NS & & & 0.009 & NS & & \\
\hline Adjuvant TACE (no versus yes) & 0.002 & 0.002 & 0.536 & $0.362-0.794$ & 0.038 & NS & & \\
\hline
\end{tabular}

Abbreviations: AFP, $a$-fetoprotein; Cl, confidential interval; GGT, $\gamma$-glutamyltransferase; HR, hazard ratio; NS, not significant; OS, overall survival; TACE, transcatheter arterial chemoembolization

Table 2 Univariate and multivariate analysis of TTR in different Cezanne expressions of HCC patients

\begin{tabular}{|c|c|c|c|c|c|c|c|c|}
\hline \multirow[t]{3}{*}{ Variables } & \multirow{3}{*}{$\frac{\text { Univariate }}{P \text {-value }}$} & \multirow{2}{*}{\multicolumn{3}{|c|}{$\begin{array}{c}\text { Low Cezanne expression } \\
\text { Multivariate }\end{array}$}} & \multirow{3}{*}{$\frac{\text { Univariate }}{P \text {-value }}$} & \multirow{2}{*}{\multicolumn{3}{|c|}{$\frac{\text { High Cezanne expression }}{\text { Multivariate }}$}} \\
\hline & & & & & & & & \\
\hline & & $P$-value & HR & $95 \% \mathrm{Cl}$ & & $P$-value & HR & $95 \% \mathrm{Cl}$ \\
\hline Gender (female versus male) & NS & NS & & & NS & NS & & \\
\hline Age, years $(\leqslant 50$ versus $>50)$ & NS & NS & & & NS & NS & & \\
\hline AFP $(\mathrm{ng} / \mathrm{ml})(\leqslant 400$ versus $>400)$ & NS & NS & & & 0.050 & NS & & \\
\hline HBsAg (negative versus positive) & 0.018 & NS & & & 0.007 & 0.019 & 0.445 & $0.226-0.874$ \\
\hline GGT $(\mathrm{U} / \mathrm{l})(\leqslant 50$ versus $>50)$ & NS & NS & & & $<0.001$ & NS & & \\
\hline Liver cirrhosis (no versus yes) & 0.001 & 0.018 & 1.767 & $1.104-2.828$ & NS & NS & & \\
\hline Tumor size $(\mathrm{cm})(\leqslant 5$ versus $>5)$ & 0.023 & 0.001 & 1.843 & $1.298-2.617$ & $<0.001$ & 0.005 & 2.218 & $1.265-3.899$ \\
\hline Tumor number (single versus multiple) & NS & NS & & & $<0.001$ & 0.023 & 2.025 & $1.103-3.718$ \\
\hline Satellite nodule (no versus yes) & NS & NS & & & 0.003 & NS & & \\
\hline Tumor capsule (no/ incomplete versus complete) & NS & NS & & & NS & NS & & \\
\hline Tumor differentiation (I-II versus III-IV) & NS & NS & & & NS & NS & & \\
\hline Vascular invasion (no versus yes) & NS & NS & & & 0.002 & NS & & \\
\hline Adjuvant TACE (no versus yes) & 0.002 & 0.002 & 0.568 & $0.397-0.812$ & $<0.001$ & NS & & \\
\hline
\end{tabular}

Abbreviations: AFP, a-fetoprotein; $\mathrm{Cl}$, confidential interval; GGT, $\gamma$-glutamyltransferase; HR, hazard ratio; NS, not significant; TACE, transcatheter arterial chemoembolization; TTR, time to recurrence

analyzed the value of Cezanne in predicting the response of adjuvant TACE in HCC.

Evidences have suggested that NF-KB signaling pathway plays an important role in various liver disease and HCC. NF- $K B$ has been related to initiation, promotion and progression of HCC. ${ }^{21,22}$ Cezanne expression was shown to be rapidly induced by NF- $\kappa \mathrm{B}$ signaling stimulated by TNF- $a$ in a negative feedback manner. ${ }^{16}$ In addition, Cezanne negatively modulates $\mathrm{NF}-\kappa \mathrm{B}$ signaling pathway that has an important role in liver pathology. ${ }^{16,23}$ Kanki et al. ${ }^{24}$ found that low Cezanne mRNA expression was correlated with poor prognosis for $\mathrm{HCC}$ patients. Moreover, Cezanne negatively regulated NF-kB signaling pathway that may be an effective target for antitumor therapy for HCC. Our previous results had demonstrated that Cezanne was downregulated in HCC tissues compared with adjacent nontumorous tissues. Moreover, Cezanne could serve as a feasible prognostic biomarker of HCC. ${ }^{17}$ Our functional studies found that short hairpin RNAs (shRNAs) against Cezanne could significantly promote tumor cell proliferation, migration and invasion. These effects were shown to be effectively inhibited when the Cezanne gene was upregulated. The role of Cezanne in inhibiting tumor metastasis was further supported by the in vivo experimental metastasis assay. Moreover, our results revealed that Cezanne expression was correlated with the expression of EMT markers. It has been known that EMT has a pivotal role in tumor invasion and metastasis in HCC, and EMT could increase the invasion of tumor cells. ${ }^{25-27}$ We therefore detected whether the effect of Cezanne on cell invasion was via induction of the EMT pathway. As expected, Cezanne expression led to increased protein and RNA levels of E-cadherin, and decreased expression of vimentin in tissue specimens. Therefore, Cezanne may inhibit HCC cells invasion via NF-kB-mediated EMT pathway, thereby contributing to prolong survival. 
a

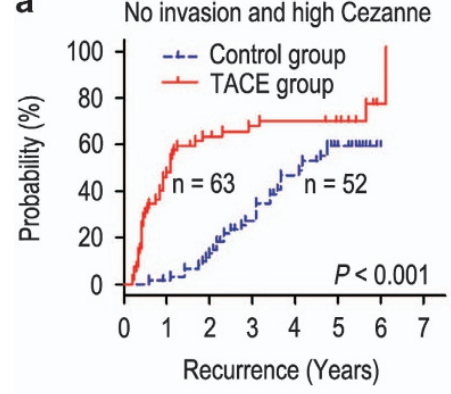

C Size $\leq 5 \mathrm{~cm}$ and high Cezanne

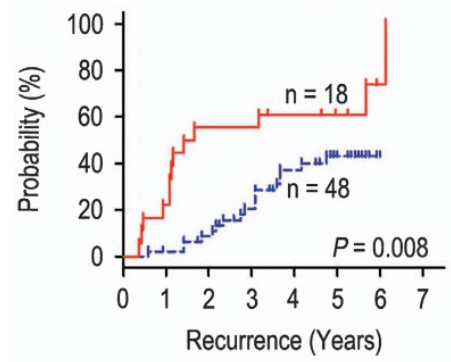

e Single tumor and high Cezanne

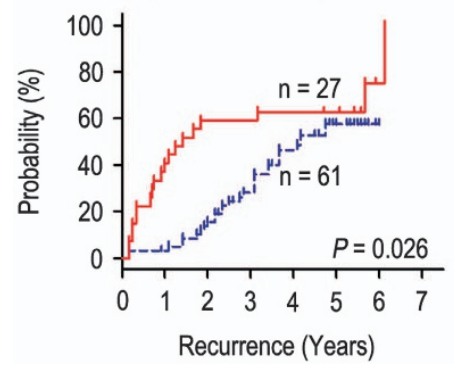

No invasion and low Cezanne

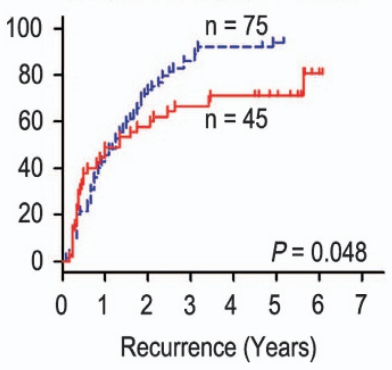

Size $\leq 5 \mathrm{~cm}$ and low Cezanne

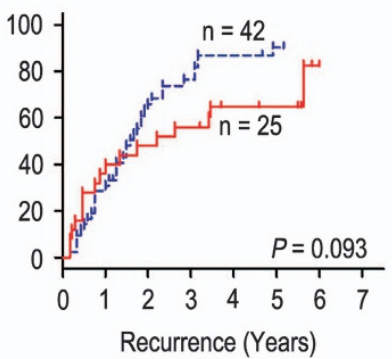

Single tumor and low Cezanne

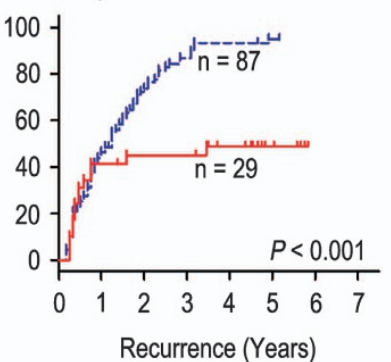

b

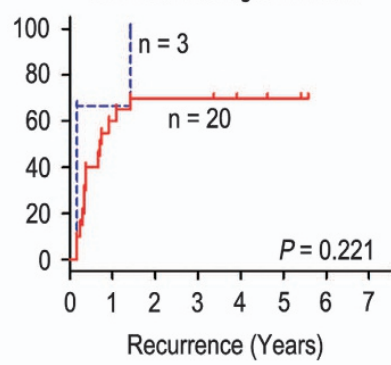

d Size $>5 \mathrm{~cm}$ and high Cezanne

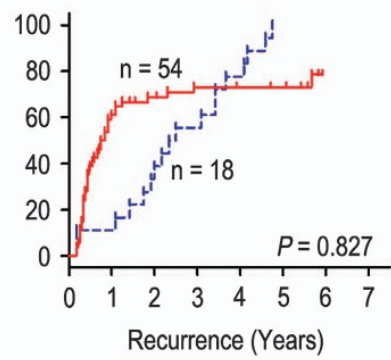

f Multiple tumor and high Cezanne

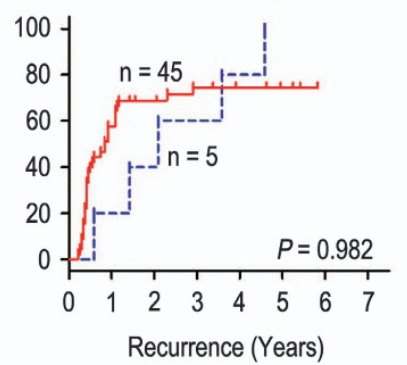

Invasion and low Cezanne

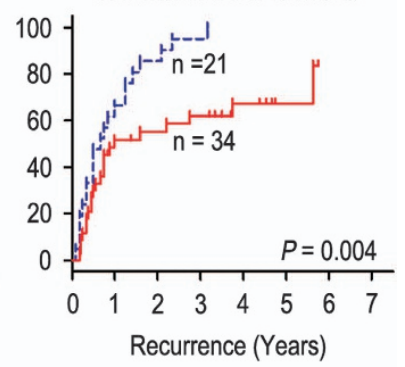

Size $>5 \mathrm{~cm}$ and low Cezanne

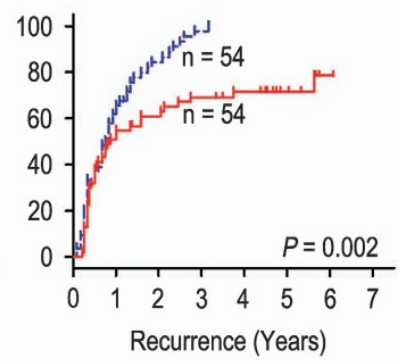

Multiple tumor and low Cezanne

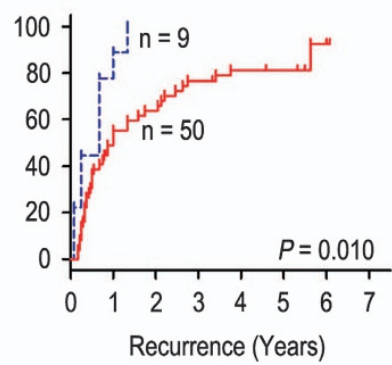

Figure 4 Cezanne predicts response to postoperative TACE in several clinical subgroups. All patients were stratified according to Cezanne levels within vascular invasion (a and $\mathbf{b}$ ), tumor size (c and $\mathbf{d}$ ) or tumor number status (e and $\mathbf{f}$ ). Kaplan-Meier survival estimates and log-rank tests were used to analyze the correlation between adjuvant TACE therapy and time to recurrence in clinical subgroups

Our results found that adjuvant TACE after surgery did not improve the prognosis of the whole study population, a similar conclusion with previous study. ${ }^{28}$ Interestingly, when the patients were stratified according to the expression of Cezanne, the outcomes of therapy with adjuvant TACE were totally different. The patients with low Cezanne expression had a favorable response to adjuvant TACE, whereas patients with high Cezanne expression had adverse response to adjuvant TACE. Moreover, in the patients with low Cezanne expression, adjuvant TACE could be an independent predictor for prognosis. It has been reported that patients with high-risk factors of recurrence, such as larger tumor size or venous invasion, are suggested to receive adjuvant TACE. ${ }^{19,20,29,30}$ However, it was also found that adjuvant TACE after resection had different outcomes of prognosis. ${ }^{31-34}$ Therefore, combining with molecular biomarkers and clinicopathologic features may help to screen patients who are suggested to receive adjuvant TACE after hepatectomy. Our data revealed that in patients with no vascular invasion, small tumor or single tumor, adjuvant TACE would shorten the TTR in high Cezanne expression patients, and prolong the TTR in low Cezanne expression patients. On the contrary, in the patients with vascular invasion, larger tumor or multiple tumor, adjuvant TACE could not provide benefits in high Cezanne expression patients, and had significant improvement in TTR in low Cezanne expression patients. Similar results were found in OS among different subgroups of $\mathrm{HCC}$ patients. These findings suggest that Cezanne status in HCC could be an important indicator in selecting patients who may benefit from adjuvant TACE to prevent relapse. Evaluating the response of adjuvant TACE after hepatectomy, based on the expression of Cezanne and clinicopathologic features, will be necessary for determining whether adjuvant TACE could be used as first-line therapy for HCC patients who have received resection.

It has been known that intrahepatic metastasis is the main reason of early recurrence. The purpose of adjuvant TACE is to kill the metastasis tumor cells in remnant liver. However, it is difficult to estimate whether the remnant liver has minimal intrahepatic metastasis or not after hepatectomy. Our results found that Cezanne was a great predictive factor for prognosis of HCC, and low Cezanne expression predicted vascular invasion and early recurrence in HCC. ${ }^{17}$ Moreover, Cezanne 
could inhibit the invasion capability of HCC cells. For patients with low Cezanne expression, postoperative adjuvant TACE could decrease the risk of early recurrence and prolong survival. However, TACE may damage remnant liver and deteriorate liver function that is possible to shorten survival of patients with high Cezanne expression who had no metastasis.

\section{Conclusion}

In this study we evaluate the role of Cezanne genes in HCC and its response to postoperative adjuvant TACE in HCC patients. Our results revealed that Cezanne may be a novel antioncogene that has a pivotal role in the invasion of $\mathrm{HCC}$ and contribute to the selection of patients who may benefit from adjuvant TACE to prevent recurrence.

\section{Materials and Methods}

Patients and specimens. The study was approved by the institutional review board and human ethics committee of Sun Yat-sen University Cancer Center. Written consent for using the samples for research purposes was obtained from all patients before surgery.

All HCC samples were collected from 313 patients who had undergone curative resections from primary HCC between January 2007 and February 2009 at the Department of Hepatobiliary Oncology, Sun Yat-sen University (Guangzhou, China) (Supplementary Table S1). There were 162 patients who only received curative resections, whereas 151 patients had undergone TACE 1-2 months after resection. The eligibility criteria of the current study were as follows: (1) all the samples were histologically confirmed, (2) none of the patients had distant metastasis or received anticancer therapies before the operation and (3) no serious complications or other malignant diseases. The cases were selected consecutively on the basis of availability of resection tissues and follow-up data. Tumor stage was classified according to the 7th edition of tumor-node-metastasis (TNM) classification of the American Joint Committee on Cancer Staging and the Barcelona Clinic Liver Cancer (BCLC) staging system. OS was defined as the date of liver resection to the date of death or last follow-up. TTR was measured from the date of surgery until the date of relapse, metastasis or last follow-up.

Cell culture. Two human HCC cell lines were used in this study: SK-Hep1 and SMCC-7721. The two cell lines were obtained from the Liver Cancer Institute of Fudan University (Shanghai, China) and routinely maintained in DMEM supplemented with $10 \%$ fetal bovine serum at $37^{\circ} \mathrm{C}$ under $5 \% \mathrm{CO}_{2}$.

IHC staining. A total of $313 \mathrm{HCC}$ tissues were used in the IHC analysis. Formalin-fixed, paraffin-embedded specimens from consenting patients were cut in $4 \mu \mathrm{m}$ sections. After being baked at $60^{\circ} \mathrm{C}$ for $2 \mathrm{~h}$, the samples were deparaffinized in xylene and rehydrated using a series of graded alcohols. Then, the tissue slides were treated with $3 \%$ hydrogen peroxide in methanol for $10 \mathrm{~min}$ to exhaust endogenous peroxidase activity. The sections were microwaved for antigen retrieval in $0.01 \mathrm{M}$ sodium citrate buffer $(\mathrm{pH} \mathrm{6.0)}$ ) for $30 \mathrm{~min}$, and then preincubated in 10\% normal goat serum for $30 \mathrm{~min}$ to prevent nonspecific staining. The sections were incubated with the Cezanne mouse monoclonal antibody (working dilution 1:200, Abcam, Cambridge, UK, ab118387), E-cadherin mouse monoclonal antibody (working dilution $1: 100$, Abcam, ab1416) and vimentin mouse monoclonal antibody (working dilution $1: 200$, Abcam, ab8978) overnight at $4^{\circ} \mathrm{C}$. The sections were treated with a nonbiotin horseradish peroxidase detection system based on the manufacturer's instructions (DAKO, Glostrup, Denmark). Assessments of the staining were scored by two experienced pathologists blinded to the patients' identity and clinical status. In discrepant cases, a pathologist reviewed the cases and reached the consensus.

Both the extent and intensity of immunostaining were taken into consideration when analyzing the data. The intensity of staining was scored from 0 to 3 , and the extent of staining was scored from 0 to $100 \%$. The final quantification of each staining was obtained by multiplying the two scores. Cezanne expression was classified as high expression if the score was higher than the median score of 1.3 , and if the score was $\leqslant 1.3$, the case was classified as low expression.
Plasmid constructs and transfection. Full-length human Cezanne cDNA was amplified by PCR and cloned into pcDNA3.1(+) expression vector (Invitrogen, Carlsbad, CA, USA), and then transfected into SMMC-7721 cell using Lipofectamine 2000 (Invitrogen) according to the manufacturer's instructions. Cell transfected with empty vector were used as controls. Stable Cezanne-expressing clones were selected by Geneticin (Rache Diagnostics, Indianapolis, IN, USA) at the concentration of $500 \mu \mathrm{g} / \mathrm{ml}$.

Establishment of Cezanne knockdown cells. Lentiviral containing shRNA targeting Cezanne was purchased from Hanbio Biotechnology Co., Ltd (Shanghai, China) and transfected into SK-Hep1 cell using Lipofectamine 2000 (Invitrogen) according to the manufacturer's instructions. Cells transfected with empty vector were used as controls. Puromycin was used to select stable clones.

Total RNA extraction and qRT-PCR. Total RNA was extracted from HCC cell lines using TRIzol reagent (Invitrogen) according to the manufacturer's protocol. The total RNA $(2 \mu \mathrm{g})$ was reverse transcribed using a PrimeScript RT Kit (Takara, Dalian, China) for first-strand CDNA synthesis. The primer sequences were: Cezanne, forward primer, $5^{\prime}$-TGGCTACCCTGGGGACTTTACTA-3', reverse primer, 5'-ACTGTCTGGGGAAGGTGGCATA-3'. GAPDH, forward primer, 5'-CTCCTCCT GTTC GACAGTCAGC-3', reverse primer, 5'-CCCAATACGACCAAATCCGTT-3'. E-cadherin, forward primer, $5^{\prime}$-CGAGAGCTACACGTTCACGG-3', reverse primer 5'-GGGTGTCGAGG GAAAAATAGG-3'. Vimentin, forward primer, 5'-GACGCCATC AACACCGAGTT-3', reverse primer, 5'-CTTTGTCGTTGGTTAGCTGGT-3'. The CDNA was subjected to quantitative real-time PCR (qRT-PCR) using the SYBR Green PCR Kit (Applied Biosystems, Carlsbad, CA, USA), and the assay was performed on an ABI PRISM 7900 Sequence Detector. 18S rRNA was used as an internal control. The relative expression level (defined as fold change) of Cezanne $\left(2^{-\triangle \triangle C t}\right)$ was normalized to the endogenous $18 \mathrm{~S}$ rRNA reference $(\triangle \mathrm{Ct})$ and related to the amount of target gene in control sample that was defined as the calibrator at 1.0 .

Western blotting. Western blot analyses were performed according to the standard protocol. The primary antibodies used for western blot are described in IHC method.

Transwell invasion assays. Invasion assay was performed with BD BioCoat Matrigel Invasion Chambers (Becton Dickinson Labware, Franklin Lakes, NJ, USA) following the manufacturer's instructions. The matrigel membrane was stained with crystal violet, and migrated cells were counted under a microscope.

Cell counting kit-8 (CCK-8) assays. Cells were cultured in 96-well plates, in which each hole contained 3000 cells and they were cultured for 24, 48, 72, 96 and $120 \mathrm{~h}$. Cells were allowed for adhesion for a period of $6 \mathrm{~h}$. Subsequently, cells were exposed to $10 \mu \mathrm{g} / \mathrm{ml}$ LPS for different durations. Once the incubation with CCK-8 (Zomanbio, Beijing, China) had been carried out for $3 \mathrm{~h}$, the corresponding optical density (OD) values were detected at $450 \mathrm{~nm}$.

Experimental metastasis assay. Two groups of 8 mice each were given intravenous tail vein injections of $1 \times 10^{6}$ shControl-SK-Hep1 cells and shCezanneSK-Hep1 cells, respectively. After 8 weeks, the mice were killed, and the tumor nodules formed on the lung surfaces were counted. The fresh lung samples were harvested and fixed with $10 \%$ formalin for histopathology analysis. Tissues were paraffin embedded and sectioned at a thickness of $5 \mu \mathrm{m}$. The sections were stained with $\mathrm{H} \& \mathrm{E}$ and then examined under the microscope to count the number of tumor nodules.

Transcatheter arterial chemoembolization. Adjuvant TACE was performed 1-2 months after hepatectomy. Hepatic arterial angiography was performed first. Among the patients without tumor stain in the remaining liver, preventive chemoembolization was done. The regimen for preventive adjuvant TACE consisted of lobaplatin $50 \mathrm{mg}$, epirubicin (EPI) $50 \mathrm{mg}$ and lipiodol $5 \mathrm{ml}$. A contrast-enhanced computed tomography (CT) was performed 1 month later, and the regimen was finished.

Follow-up. The last follow-up was February 2015. Recurrence was confirmed by serum $\alpha$-fetoprotein (AFP) level, abdominal ultrasonography every 2 months and CT or magnetic resonance imaging or positron emission tomography every 6 months 
after hepatectomy. The main causes of death were HCC recurrence or complicated cirrhosis of the liver.

Statistical analysis. The SPSS software package (version 16.0, Chicago, IL, USA) was used for the statistical analysis. The mRNA levels of Cezanne, E-cadherin and vimentin in HCC cells were compared using paired Student's t-test. Pearson's $\chi^{2}$ test was applied to analyze the correlation of Cezanne with E-cadherin and vimentin staining scores. Survival curves were generated using the KaplanMeier method, and differences between curves were estimated by the log-rank test. All $P$-values were two sided and $P$-values of ${ }^{<} 0.05$ were considered to be statistically significant.

\section{Conflict of Interest}

The authors declare no conflict of interest.

Acknowledgements. We gratefully acknowledge Limin Zheng for the help in our experiment (State Key Laboratory of Oncology in South China, Cancer Center, Sun Yat-sen University). This work was supported by a grant of National Natural Science Foundation of China (№. 81172037).

\section{Author contributions}

J-HW, R-PG and WW participated in the study conception, design, case selection and experiments. J-HW, X-PZ and Y-FZ carried out the data collection. X-LW, S-HL and MS performed experiment in vitro and in vivo. $\mathrm{P}-\mathrm{EJ}$ and $\mathrm{Y}-\mathrm{HL}$ performed the scoring of immunohistochemical staining. R-PG, M-SC and J-HW performed the data analysis and writing of the manuscript. All the authors read and approved the final manuscript.

\section{Publisher's Note}

Springer Nature remains neutral with regard to jurisdictional claims in published maps and institutional affiliations.

1. Jemal A, Bray F, Center MM, Ferlay J, Ward E, Forman D. Global cancer statistics. $C A$ Cancer J Clin 2011; 61: 69-90.

2. El-Serag HB, Rudolph KL. Hepatocellular carcinoma: epidemiology and molecular carcinogenesis. Gastroenterology 2007; 132: 2557-2576.

3. El-Serag HB. Hepatocellular carcinoma. N Engl J Med 2011; 365: 1118-1127.

4. Tsai TJ, Chau GY, Lui WY, Tsay SH, King KL, Loong CC et al. Clinical significance of microscopic tumor venous invasion in patients with resectable hepatocellular carcinoma. Surgery 2000; 127: 603-608.

5. Zhu AX. Systemic therapy of advanced hepatocellular carcinoma: how hopeful should we be? Oncologist 2006; 11: 790-800.

6. Yeo W, Mok TS, Zee B, Leung TW, Lai PB, Lau WY et al. A randomized phase III study of doxorubicin versus cisplatin/interferon alpha-2b/doxorubicin/fluorouracil (PIAF) combination chemotherapy for unresectable hepatocellular carcinoma. J Natl Cancer Inst 2005; 97 1532-1538.

7. Leung TW, Patt YZ, Lau WY, Ho SK, Yu SC, Chan AT et al. Complete pathological remission is possible with systemic combination chemotherapy for inoperable hepatocellular carcinoma. Clin Cancer Res 1999; 5: 1676-1681.

8. Imamura H, Matsuyama Y, Tanaka E, Ohkubo T, Hasegawa K, Miyagawa $S$ et al. Risk factors contributing to early and late phase intrahepatic recurrence of hepatocellular carcinoma after hepatectomy. J Hepatol 2003; 38: 200-207.

9. Li KW, Li X, Wen TF, Lu WS. The effect of postoperative TACE on prognosis of HCC: an update. Hepatogastroenterology 2013; 60: 248-251.

10. Poon RT, Fan ST, Lo CM, Liu CL, Ng IO, Wong J. Long-term prognosis after resection of hepatocellular carcinoma associated with hepatitis B-related cirrhosis. J Clin Oncol 2000; 18 : 1094-1101.

11. Sieghart W, Hucke F, Pinter M, Graziadei I, Vogel W, Muller C et al. The ART of decision making: retreatment with transarterial chemoembolization in patients with hepatocellular carcinoma. Hepatology 2013; 57: 2261-2273.

12. Aravalli RN, Steer CJ, Cressman EN. Molecular mechanisms of hepatocellular carcinoma Hepatology 2008; 48: 2047-2063.

13. Dragani TA. Risk of HCC: genetic heterogeneity and complex genetics. J Hepatol 2010; 52 252-257.
14. Marquardt JU, Galle PR, Teufel A. Molecular diagnosis and therapy of hepatocellular carcinoma (HCC): an emerging field for advanced technologies. J Hepatol 2012; 56: 267-275.

15. Evans PC, Smith TS, Lai MJ, Williams MG, Burke DF, Heyninck $K$ et al. A novel type of deubiquitinating enzyme. J Biol Chem 2003; 278: 23180-23186.

16. Enesa K, Zakkar M, Chaudhury H, Luong le A, Rawlinson L, Mason JC et al. NF-kappaB suppression by the deubiquitinating enzyme Cezanne: a novel negative feedback loop in proinflammatory signaling. J Biol Chem 2008; 283: 7036-7045.

17. Wang JH, Wei W, Guo ZX, Shi M, Guo RP. Decreased Cezanne expression is associated with the progression and poor prognosis in hepatocellular carcinoma. J Transl Med 2015; 13: 41.

18. Ouchi K, Sugawara T, Fujiya T, Kamiyama $\mathrm{Y}$, Kakugawa $\mathrm{Y}$, Mikuni $\mathrm{J}$ et al. Prediction of recurrence and extratumor spread of hepatocellular carcinoma following resection. J Surg Oncol 2000; 75: 241-245.

19. Ren ZG, Lin ZY, Xia JL, Ye SL, Ma ZC, Ye QH et al. Postoperative adjuvant arterial chemoembolization improves survival of hepatocellular carcinoma patients with risk factors for residual tumor: a retrospective control study. World J Gastroenterol 2004; 10: 2791-2794.

20. Peng BG, He Q, Li JP, Zhou F. Adjuvant transcatheter arterial chemoembolization improves efficacy of hepatectomy for patients with hepatocellular carcinoma and portal vein tumor thrombus. Am J Surg 2009; 198: 313-318.

21. Arsura M, Cavin LG. Nuclear factor-kappaB and liver carcinogenesis. Cancer Lett 2005; 229: 157-169.

22. Maeda S, Omata M. Inflammation and cancer: role of nuclear factor-kappaB activation. Cancer Sci 2008; 99: 836-842.

23. Sun B, Karin M. NF-kappaB signaling, liver disease and hepatoprotective agents. Oncogene 2008; 27: 6228-6244.

24. Kanki K, Akechi $Y$, Ueda $\mathrm{C}$, Tsuchiya $\mathrm{H}$, Shimizu $\mathrm{H}$, Ishijima $\mathrm{N}$ et al. Biological and clinical implications of retinoic acid-responsive genes in human hepatocellular carcinoma cells. $J$ Hepatol 2013; 59: 1037-1044.

25. Arias AM. Epithelial mesenchymal interactions in cancer and development. Cell 2001; 105 425-431.

26. Thiery JP. Epithelial-mesenchymal transitions in tumour progression. Nat Rev Cancer 2002; 2: 442-454.

27. Zhang Q, Bai X, Chen W, Ma T, Hu Q, Liang C et al. Wnt/beta-catenin signaling enhances hypoxia-induced epithelial-mesenchymal transition in hepatocellular carcinoma via crosstalk with hif-1alpha signaling. Carcinogenesis 2013; 34: 962-973.

28. Lencioni R. Loco-regional treatment of hepatocellular carcinoma. Hepatology 2010; 52 : 762-773.

29. Xi T, Lai EC, Min AR, Shi LH, Wu D, Xue F et al. Adjuvant transarterial chemoembolization after curative resection of hepatocellular carcinoma: a non-randomized comparative study. Hepatogastroenterology 2012; 59: 1198-1203.

30. Huang L, Li J, Yan J, Cao J, Liu C, Zhang X et al. Early recurrence after curative resection in oligonodular hepatocellular carcinoma. Hepatogastroenterology 2013; 60: 28-31.

31. Ono T, Nagasue N, Kohno H, Hayashi T, Uchida M, Yukaya H et al. Adjuvant chemotherapy with epirubicin and carmofur after radical resection of hepatocellular carcinoma: a prospective randomized study. Semin Oncol 1997; 24(2 Suppl 6): S6-18-S16-25.

32. Ono T, Yamanoi A, Nazmy El Assal O, Kohno H, Nagasue N. Adjuvant chemotherapy after resection of hepatocellular carcinoma causes deterioration of long-term prognosis in cirrhotic patients: metaanalysis of three randomized controlled trials. Cancer 2001; 91: 2378-2385.

33. Lai EC, Lo CM, Fan ST, Liu CL, Wong J. Postoperative adjuvant chemotherapy after curative resection of hepatocellular carcinoma: a randomized controlled trial. Arch Surg 1998; 133: 183-188.

34. Schwartz JD, Schwartz M, Mandeli J, Sung M. Neoadjuvant and adjuvant therapy for resectable hepatocellular carcinoma: review of the randomised clinical trials. Lancet Oncol 2002; 3: 593-603.

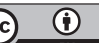

Cell Death and Disease is an open-access journal published by Nature Publishing Group. This work is licensed under a Creative Commons Attribution 4.0 International License. The images or other third party material in this article are included in the article's Creative Commons license, unless indicated otherwise in the credit line; if the material is not included under the Creative Commons license, users will need to obtain permission from the license holder to reproduce the material. To view a copy of this license, visit http://creativecommons.org/licenses/by/4.0/

(C) The Author(s) 2017 